\title{
Implante de válvula de Ahmed vía pars plana para el manejo del glaucoma uveítico refractario
}

\section{Placement of Ahmed Valve through the pars plana for the management of refractory uveitic glaucoma}

Correspondencia Fernando Munayco Guillen fernandomg345@hotmail.com

Recibido: 27/03/2018

Arbitrado por pares

Aprobado: 20/06/2018

Citar como: Munayco-Guillén F, Valderrama-Albino V, CamposDávila B, Loayza-Gamboa W, Bauman-Rivera K, MalaverSandoval D. Implante de válvula de Ahmed vía pars plana para el manejo del glaucoma uveítico refractario. Acta Med Peru. 2018;35(3):180-3

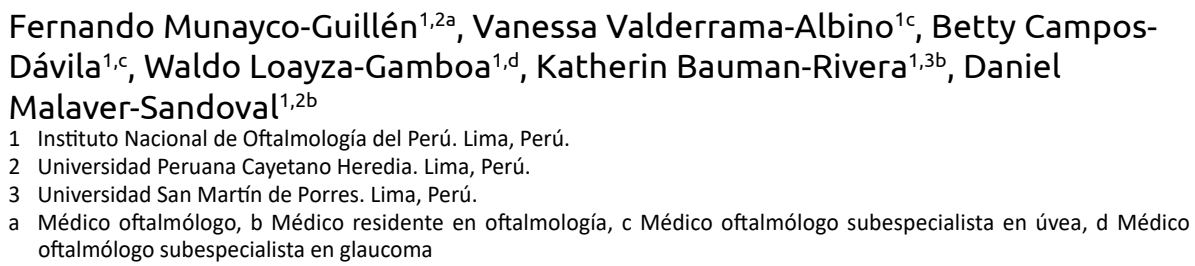

\section{RESUMEN}

La uveítis es la tercera causa de ceguera evitable, siendo una de sus complicaciones el glaucoma uveítico. Presentamos el caso de una paciente de 25 años tratada con extracción extra capsular de cristalino y trabeculectomía AO, con posterior ciclocrioterapia bilateral, llegando a controlar la enfermedad inflamatoria de fondo y presión intraocular (PIO) en el ojo derecho por siete años con posterior pérdida de la visión (pthisis bulbi). El ojo izquierdo se mantuvo estable por 15 años, presentando luego una PIO de $50 \mathrm{mmHg}$, por lo que se implanta una válvula de Ahmed por vía pars plana con previa vitrectomía, debido a las alteraciones anatómicas del segmento anterior. Se controló la PIO y se obtuvo una visión de 20/70 con correctores, sin uso de antihipertensivo ocular. El manejo adecuado de la enfermedad de fondo y el uso de dispositivos de drenaje valvulados son la mejor opción para el glaucoma uveítico. Una gran alternativa de abordaje es el implante vía pars plana, previa vitrectomía.

Palabras clave: Glaucoma; Uveítis; Coroiditis; Vitrectomía (fuente: DeCS BIREME).

\section{ABSTRACT}

Uveitis is the third preventable cause of blindness, and uveitic glaucoma is one of its complications. We present the case of a 25-year old female patient who underwent an extracapsular lens excision and $A O$ trabeculectomy, and afterwards bilateral cyclocryotherapy was performed. The underlying inflammatory condition was controlled, and the intraocular pressure (IOP) in the right eye was also controlled for seven years, although she later lost sight (pthisis bulbi). The left eye remained in a stable condition for 15 years, but afterwards it developed $50 \mathrm{~mm} \mathrm{Hg} \mathrm{IOP,} \mathrm{so} \mathrm{an} \mathrm{Ahmed} \mathrm{Valve} \mathrm{through} \mathrm{the} \mathrm{pars} \mathrm{plana}$ was placed, after having performed a vitrectomy because of the anatomic alterations in the anterior segment. The IOP got controlled and 20/70 vision using glasses was attained, without the use of an ocular antihypertensive compound. Adequate management of the underlying disease and the use of valve drainage systems constitute the best choice for the management of uveitic glaucoma. A good approach option is placing the device through the pars plana, after having performed a vitrectomy.

Keywords: Glaucoma; Uveitis; Choroiditis; Vitrectomy (source: MeSH NLM). 


\section{INTRODUCCIÓN}

La uveítis es la tercera causa de ceguera evitable, siendo más frecuente en mujeres. Una de sus peores complicaciones por la dificultad en su manejo terapéutico es el glaucoma uveítico (GU) ${ }^{[1]}$. El GU fue descrito por primera vez por Joseph Beer en $1813^{[1,2]}$, y se estima que un $20 \%$ a $40 \%$ de pacientes con uveítis desarrollan GU $(6,5 \%$ al año, $11,1 \%$ a los cinco años y $22,7 \%$ a los diez años) ${ }^{[1,3,4]}$.

Dentro de las causas más frecuentes de GU se señala a la ciclitis heterocrómica de Fuch (59\%), uveítis herpética (54\%), oftalmía simpática (43\%), síndrome de Vogt Koyanagi Harada (38\%), síndrome de Possner Scholssman (25\%), coroiditis multifocal $(27 \%)$ y uveítis artrítica $(21 \%)^{[1,2,5]}$.

Respecto a su fisiopatología, el GU se produce por un desequilibrio entre la producción y la resistencia a la salida del humor acuoso. Al inicio de la enfermedad hay hiposecreción de humor acuoso por la inflamación y mayor salida por la vía uveoescleral. La inflamación produce un gran estrés oxidativo, disminución de la permeabilidad de la barrera hematoacuosa y obstrucción mecánica de la malla trabecular, producida por la acumulación de células inflamatorias, proteínas y fibrina ${ }^{[1,2,3]}$. El GU puede ser de ángulo abierto o cerrado, siendo el de tipo abierto el más frecuente ${ }^{[1,2]}$.

Muchos pacientes con GU no controlarán adecuadamente la presión intraocular con terapia médica y serán sometidos a cirugía. Dentro de las principales técnicas quirúrgicas para el manejo del glaucoma uveítico tenemos a la trabeculectomía, uso de dispositivos de drenaje (valvulados o no valvulados). También se tiene alternativas como: la ciclofotocoagulación y ciclocrioterapia ${ }^{[5]}$.

El objetivo de este caso es presentar la evolución de la enfermedad, el manejo clínico y quirúrgico de un paciente con GU, ojo único y de pronósticos visual y anatómico muy reservados, haciendo uso de un dispositivo de drenaje con abordaje por vía pars plana.

\section{REPORTE DE CASO}

Paciente de 25 años (en el año 1999) de sexo femenino acude a nuestro instituto por presentar disminución progresiva de agudeza visual (AV), ojo rojo y dolor tipo opresivo moderado en ambos ojos (AO). Presenta un tiempo de enfermedad de un mes, de inicio insidioso y curso progresivo. Presenta AV inicial de movimiento de manos en $\mathrm{AO}$ y presión intraocular de 31 y $37 \mathrm{mmHg}$. En cámara anterior se observa signos de una uveítis anterior de tipo granulomatoso bilateral con Tyndall y flare $(+++)$, precipitados retroqueráticos gruesos, catarata uveítica en $\mathrm{AO}$ con sinequias posteriores (fondo de ojo no evaluable en AO). En la superficie ocular presentaba pterigión nasotemporal bilateral de II grado. Se le prescribió tratamiento con cuatro drogas hipotensoras más atropina y prednisolona, pero no tuvo respuesta adecuada al tratamiento.
Se controla la inflamación y tres meses después se le realiza una cirugía de extracción extracapsular de catarata más trabeculectomía con mitomicina e iridotomía periférica quirúrgica en $\mathrm{AO}$, con diferencia de un mes entre ambas cirugías, quedando en afaquia bilateral.

Posterior a la cirugía, tiene una presión intraocular (PIO) promedio de $17 \mathrm{mmHg}$ AO. Al mes de la cirugía presenta reactivación de la uveítis e incremento de la PIO en el ojo izquierdo (OI) llegando hasta $27 \mathrm{mmHg}$, siendo controlado con hipotensores tópicos por dos meses y se decide aplicarle ciclocrioterapia en $180^{\circ}$ inferior en el OI, manteniendo la PIO estable por un tiempo de dos años.

Se llega al diagnóstico clínico de coroiditis multifocal con panuveítis, al evidenciarse lesiones cicatrizales periféricas en Ol y por contar con una ecografía ocular que presenta $2,66 \mathrm{~mm}$ de grosor coroideo y opacidad vítrea moderada, observándose excavación papilar visible.

El diagnóstico diferencial se realizó entre patologías que podían tener como característica la panuveítis bilateral y glaucoma uveítico: tuberculosis ocular, sífilis ocular, síndrome de Vogt Koyanagi Harada, sarcoidosis; quedaron descartados porque la clínica no era concordante y los exámenes de laboratorio e imágenes eran negativos.

En el ojo derecho (OD) después de la trabeculectomía, la PIO se mantuvo estable por dos años, pero se volvió a incrementar, motivo por el cual se somete a ciclocrioterapia a $180^{\circ}$ inferior. Se inicia tratamiento con ciclofosfamida $50 \mathrm{mg}$ y metotrexato $10 \mathrm{mg}$ y se mantuvo con este tratamiento por tres años y medio. En este periodo la PIO se mantiene estable en AO. Se suspende la ciclofosfamida y metotrexate y se inicia el tratamiento con azatioprina $50 \mathrm{mg}$.

Se controla adecuadamente la PIO en el OI, pero el OD luego de siete años (posterior a su primera cirugía) entra en pthisis bulbi. El OI desarrolla queratopatía en banda, motivo por el cual no era posible evaluar adecuadamente el nervio óptico y el polo

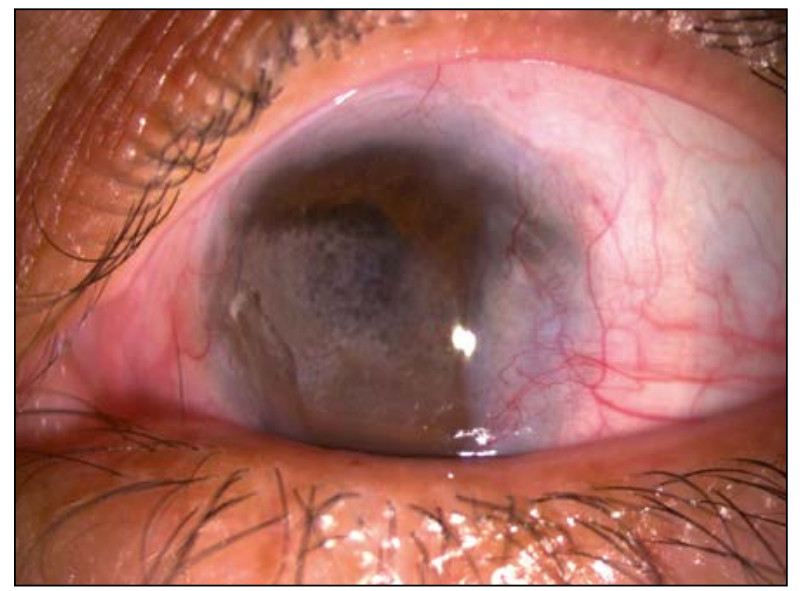

Figura 1. Ojo izquierdo en afaquia que muestra queratopatía en banda antes de la cirugía. 


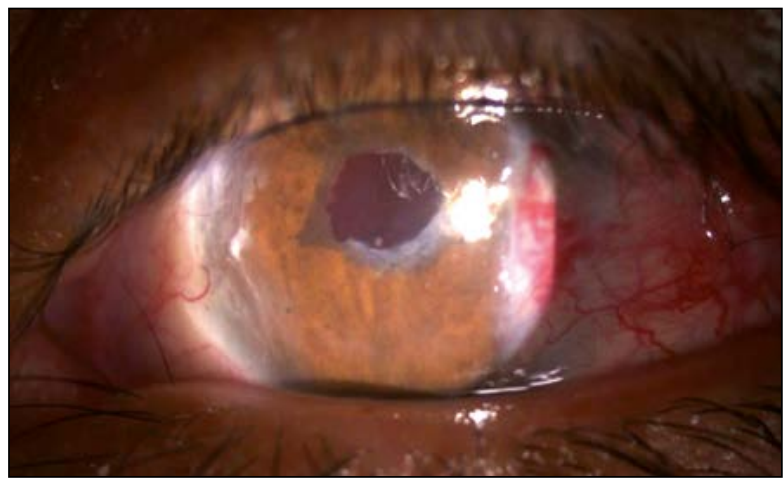

Figura 2. Ojo izquierdo en afaquia después de la cirugía. No se observa la queratopatía en banda.

posterior (ver Figura 1). La PIO se mantiene estable por unos 15 años en el OI, pero luego de este periodo, esta aumenta a 50 $\mathrm{mmHg}$ y presenta una AV de 20/400. Se utiliza como tratamiento inmunosupresor metotrexato $10 \mathrm{mg}$ y se instaura terapia antiglaucomatosa máxima, pero no se logra controlar la PIO.

Ante esta situación se decide colocar un dispositivo de drenaje, pero se tenía el inconveniente que el segmento anterior estaba seriamente dañado debido a las secuelas de la inflamación intensa, por lo que se tenía sinequias anteriores (entre la córnea y el iris) y sinequias en la malla trabecular. Además, presentaba un recuento endotelial bajo de $1450 \mathrm{cel} / \mathrm{um}^{2}$, lo que dificultaba implantar el tubo de la válvula en el segmento anterior. Por tal motivo, se buscó otra alternativa de abordaje, por lo que, en junta médica, se decide implantar el tubo de la válvula por vía pars, previa vitrectomía. Durante la cirugía se remueve mecánicamente la queratopatía en banda, con la finalidad de mejorar la visibilidad de medios para la cirugía del implante de válvula.

A los tres meses se obtiene una AV de 20/70 con correctores y una PIO de $13 \mathrm{mmHg}$, con la posterior suspensión de drogas antiglaucomatosas. Se suspende el metotrexato y se reinicia tratamiento con azatioprina de $75 \mathrm{mg}$ y diclofenaco en gotas (ver Figura 2).

\section{DISCUSIÓN}

La coroiditis multifocal con panuveítis, es una uveítis crónica, bilateral e idiopática, caracterizada por múltiples lesiones coriorretinianas blanco amarillentas periféricas en sacabocado (estrías de Schlegel), celularidad moderada en cámara anterior $(50 \%)$ y vitreítis moderada a severa $(98 \%)$. Se presenta más frecuentemente en pacientes miopes de sexo femenino y de raza blanca (80\%). No se asocia a ninguna enfermedad sistémica ni ocular, por lo que se le considera un diagnóstico de exclusión. Dentro de sus complicaciones más frecuentes se encuentra: catarata (40\%), neovascularización coroidea (30\%), edema macular cistoide (30\%) y glaucoma uveítico $(10 \%)^{[1-4]}$. El caso presentado tuvo catarata y glaucoma uveítico como complicaciones, siendo esta última la de más difícil manejo. La paciente fue sometida a tratamiento médico y a múltiples cirugías para llegar a un control aceptable de la presión intraocular, debido a la naturaleza refractaria de este tipo glaucoma.

Para la terapia médica antihipertensiva ocular, se puede usar los betabloqueadores y los alfa agonistas, tratando de evitar los análogos de prostanglandinas, por su posible incremento del edema macular cistoide y la iridociclitis. También se debe evitar el uso de inhibidores de la anhidrasa carbónica porque pueden ocasionar edema corneal y disminución de recuento endotelial ${ }^{[3]}$.

Se debe tener en cuenta que, para el manejo de la etapa aguda de la inflamación de la coroiditis multifocal con panuveítis, se usan corticoides de manera frecuente. Se describe que hasta un tercio de los pacientes pueden presentar hipertensión ocular secundaria a corticoides entre las dos a seis semanas de uso. Esto se debe al acúmulo de glucosaminoglucanos a nivel trabecular e inhibición de la fagocitosis de las células endoteliales de la malla trabecular y cambios en el citoesqueleto ${ }^{[1,3]}$. Se recomienda como alternativa de uso a los inmunomoduladores, los cuales permiten un ahorro del uso de corticoides y evitan las complicaciones hasta un $83 \%$, reduciendo el riesgo de pérdida visual, ya que el curso de la coroiditis multifocal es crónica ${ }^{[5]}$.

La trabeculectomía con mitomicina, ha demostrado que tiene una baja tasa de éxito en el tiempo: $90 \%$ de éxito al primer año, pero disminuye al $30 \%$ de éxito a los 5 años ${ }^{[1,3,6]}$. Se considera que los dispositivos de drenaje valvulados (válvula de Ahmed), son de elección para el manejo del glaucoma uveítico con una tasa de éxito de $87 \%$ a $94 \%$ a los 5 años ${ }^{[7,8]}$.

El caso reportado presentaba alteraciones anatómicas en el segmento anterior (sinequias anteriores y posteriores, córnea con bajo recuento endotelial) (ver Figura 3), por lo cual se consideró como una alternativa de tratamiento al implante de válvula de Ahmed por vía pars plana, con previa vitrectomía, para que el tubo valvular quede en cavidad vítrea. Las indicaciones de este abordaje son: alteraciones en el segmento anterior, cámara anterior poco profunda, recuento endotelial bajo (alto riesgo de descompensación corneal) ${ }^{[9,10]}$.

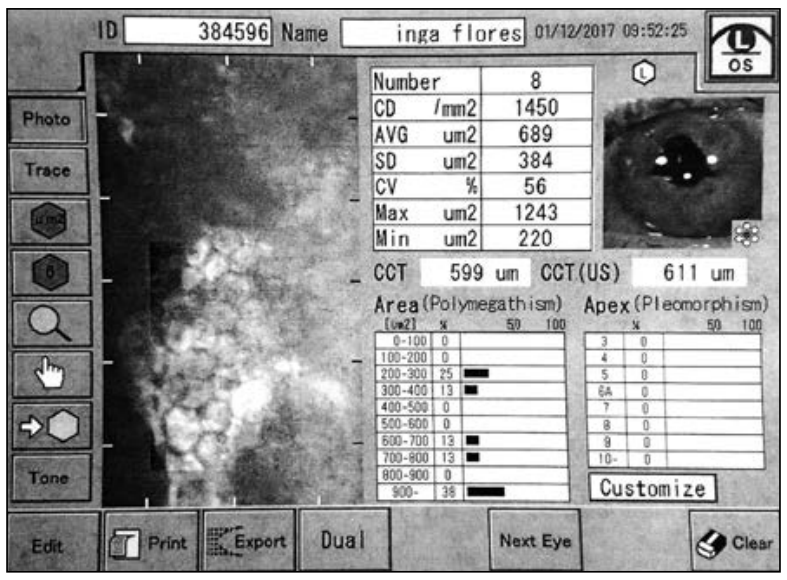

Figura 3. Recuento endotelial bajo con polimegatismo aumentado. 
Se ha reportado en la literatura, como complicaciones de este abordaje: oclusión del tubo, descompensación corneal, diplopía, extrusión del tubo, hipotonía severa, maculopatía hipotónica, desprendimiento de retina, desprendimiento coroideo hemorrágico ${ }^{[3,9,11]}$.

Se comparó la conservación de las células endoteliales del implante de válvula de Ahmed vía cámara anterior versus pars plana y se encontró que se pierde menos células endoteliales en el abordaje posterior, con una pérdida similar a una cirugía de catarata ${ }^{[9]}$.

Nuestro paciente comenzó su enfermedad hace aproximadamente hace 18 años (1999), por lo cual su tratamiento estuvo supeditado a la evidencia científica que se contaba por esos años, su condición socioeconómica y a la disponibilidad de medios terapéuticos que teníamos en nuestra institución. El primer dispositivo de drenaje, se usó en el año 1991 para el manejo del glaucoma neovascular ${ }^{[12]}$. Debido al alto costo y a la accesibilidad de estos dispositivos en nuestro país por esos años, no se pudo utilizar, pero se logró estabilizar la PIO a través de la ciclocrioterapia, pero a pesar de ello el ojo derecho se perdió. Luego de estar 15 años estable, la paciente vuelve a descompensar su presión en el Ol y se es indispensable el uso de este dispositivo. El Seguro Integral de Salud (SIS) (seguro ofrecido por el Estado Peruano), cobertura actualmente los gastos de la válvula, lo cual permitió su uso en nuestro paciente.

Otras terapias alternativas para el tratamiento para el glaucoma uveítico es la ciclofotocoagulación que actualmente se recomienda como último recurso cuando hay fracaso de otras terapias médica y quirúrgica. Tiene una tasa de éxito de $72,2 \%$ y una tasa de complicaciones de 7,1\% como: pthisis bulbi (peor complicación: 0,8\%), edema macular cistoide, hifema, oftalmía simpática, catarata secundaria, quemaduras ${ }^{[13,14]}$.

Concluimos que el implante de válvula de Ahmed vía pars plana con previa vitrectomía, puede preservar la visión, disminuir la presión intraocular y disminuir el número de fármacos hipotensores oculares. El abordaje por vía pars plana se constituye como una gran alternativa de manejo, en casos de glaucomas uveíticos donde no se pueda implantar el tubo valvular en el segmento anterior.
Fuentes de financiamiento: autofinanciado.

Conflictos de interés: los autores declaran no tener ningún conflicto de intereses.

\section{REFERENCIAS BIBLIOGRÁFICAS}

1. Siddique SS, Suelves AM, Baheti U, Foster CS. Glaucoma and uveítis. Surv Ophthalmol. 2013;58(1):1-10.

2. Baneke AJ, Lim KS, Stanford M. The pathogenesis of raised intraocular pressure in uveitis. Curr Eye Res. 2016;41(2):137-49.

3. Sng CC, Ang M, Barton K. Uveitis and glaucoma: new insights in the pathogenesis and treatment. Prog Brain Res. 2015;221:243-69.

4. Shimizu A, Maruyama K, Yokoyama Y, Tsuda S, Ryu M, Nakazawa T. Characteristics of uveitic glaucoma and evaluation of its surgical treatment. Clin Ophthalmol. 2014;26(8):2383-9.

5. Sayed M, Lee R. Current management approaches for uveitic glaucoma. Int Ophthalmol Clin. 2015;55(3):141-60.

6. Da Mata A, Burk SE, Netland PA, Baltatzis S, Christen W, Foster CS. Management of uveitic glaucoma with Ahmed glaucoma valve implantation. Ophthalmology. 1999;106(11):2168-72.

7. Papadaki TG, Zacharopoulos IP, Pasquale LR, Christen WB, Netland PA, Foster CS. Long-term results of Ahmed glaucoma valve implantation for uveitic glaucoma. Am J Ophthalmol. 2007;144(1):62-9.

8. Bettis DI, Morshedi RG, Chaya C, Goldsmith J, Crandall A, Zabriskie $\mathrm{N}$. Trabeculectomy with mitomycin Cor Ahmed valve implantation in eyes with uveitic glaucoma. J Glaucoma. 2015;24(8):591-9.

9. Chihara E, Umemoto $M$, Tanito $M$. Preservation of corneal endothelium after pars plana tube insertion of the Ahmed glaucoma valve. Jpn J Ophthalmol. 2012;56(2):119-27.

10. Maris PJ Jr, Tsai JC, Khatib N, Bansal R, Al-Aswad LA. Clinical outcomes of Ahmed glaucoma valve in posterior segment versus anterior chamber. .J Glaucoma. 2013;22(3):183-9.

11. Sungur G, Yakin M, Eksioglu U, Satana B, Ornek F. Assessment of conditions affecting surgical success of Ahmed glaucoma valve implants in glaucoma secondary to different uveítis etiologies in adults. Eye (Lond). 2017;31(10):1435-42.

12. Maris PJ Jr, Tsai JC, Khatib N, Bansal R, Al-Aswad LA. Clinical outcomes of Ahmed glaucoma valve in posterior segment versus anterior chamber. J Glaucoma. 2013;22(3):183-9.

13. Cheung JJC, Li KKW, Tang SWK. Retrospective review on the outcome and safety of transscleral diode laser cyclophotocoagulation in refractory glaucoma in Chinese patients. Int Ophthalmol. 2017. doi: 10.1007/s10792-017-0782-1.

14. Ishida K. Update on results and complications of cyclophotocoagulation. Curr Opin Ophthalmol. 2013;24(2):102-10. 